\section{Cureus}

Received 07/16/2018

Review began 09/15/2018

Review ended 03/25/2019

Published 04/03/2019

\section{(c) Copyright 2019}

Loriaux et al. This is an open access article distributed under the terms of the Creative Commons Attribution License CC-BY 3.0., which permits unrestricted use, distribution, and reproduction in any medium, provided the original author and source are credited.

\title{
Ethylene Glycol Toxicity in the Setting of Recurrent Ingestion: A Case Report and Literature Review
}

\author{
Daniel Loriaux ${ }^{1}$, Stephen P. Bergin ${ }^{2}$, Sweta M. Patel ${ }^{2}$, Jesse Tucker ${ }^{2}$, Christina E. \\ Barkauskas $^{2}$ \\ 1. Internal Medicine, Brigham \& Women's Hospital, Harvard Medical School, Boston, USA 2. Internal \\ Medicine - Critical Care, Duke University Medical Center, Durham, USA
}

$\square$ Corresponding author: Daniel Loriaux, dloriaux@bwh.harvard.edu Disclosures can be found in Additional Information at the end of the article

\section{Abstract}

Ethylene glycol (EG) poisoning is a toxicologic emergency requiring high clinical suspicion and early diagnosis to prevent life-threatening complications. Direct EG quantification methods involve cumbersome and time-consuming laboratory tests of limited utility in the emergency setting. Accordingly, the osmolal gap is frequently employed as a surrogate screening method in cases of suspected toxic alcohol poisoning. However, the osmolal gap has several inherent limitations to be considered when used as a diagnostic tool for EG toxicity. Although many of these limitations are widely acknowledged, the clinical finding of a normal serum osmolal gap in the setting of recurrent toxic alcohol exposure is an observation that has remained largely unexplored. The purpose of this case report is to characterize the accelerated osmolal gap to anion gap conversion that may occur in the setting of chronic toxic alcohol abuse.

Categories: Emergency Medicine, Internal Medicine

Keywords: ethylene glycol toxicity, osmolal gap

\section{Introduction}

Ethylene glycol (EG) is an odorless, electrically neutral polyalcohol and a common solvent for antifreeze solutions, brake fluids, and household industrial products [1-2]. The sweet taste, low cost, and intoxicating effects of EG lead to its illicit use as an ethanol substitute [2-3]. In cases of both accidental and purposeful ingestion, timely diagnosis of EG toxicity enables the administration of agents that block the formation of toxic metabolites (ethanol or fomepizole) or initiation of hemodialysis to avoid systemic, irreversible, and life-threatening complications [4-6]. However, early diagnosis and intervention is challenging for several reasons. The initial clinical presentation is nonspecific, involving a constellation of symptoms including altered sensorium, headache, slurred speech, ataxia, nystagmus, nausea, and vomiting. When EG ingestion is suspected, direct serum assays are limited in availability and many institutions must resort to send-out laboratory testing [7]. When direct detection methods are available, the prolonged turnaround time to perform the necessary diagnostic studies precludes direct screening in the emergency setting [8]. The osmolal gap is a substitute indicator widely regarded as the best available screening test for toxic alcohol ingestion [9-10].

Although commonly utilized, the osmolal gap lacks sensitivity. This is due to the fact that EG (an uncharged, osmotically active parent alcohol) is oxidized by alcohol dehydrogenase (ADH) into toxic metabolites that are negatively charged at physiological $\mathrm{pH}$. 
These metabolites exist in a dissociated state and are accompanied by a cation (sodium), which makes them undetectable in the osmolal gap [6]. Diagnostically this manifests as the disappearance of the osmolal gap in exchange for a rising serum anion gap and an artefactual lactic acidosis, which is due to the cross-reaction of the EG metabolites with L-lactate oxidase in blood gas analyzers and lactate electrodes [11].

During EG metabolism, the transition point between the elevated osmolal gap and anion gap is largely dependent on the enzymatic activity of $\mathrm{ADH}$, which is influenced by age, gender, body mass, nutrition, exercise, and prior exposure to alcohol [12]. Similar to the enhanced ADH activity that is observed in chronic alcoholism, in vivo research shows that the efficiency of $\mathrm{ADH}$ in metabolizing toxic alcohols is amplified by chronic exposure [13]. This is clinically significant because the increased metabolic efficiency of ADH in the setting of chronic toxic alcohol abuse may significantly shorten the duration an elevated osmolal gap remains detectable. The case presented in this report highlights the clinical importance of accelerated EG metabolism and the potentially misleading nature of lactic acidosis when both the osmolal gap and toxicology studies are found to be normal.

\section{Case Presentation}

A 38-year-old woman with a past medical history of chronic alcohol abuse, seizures, and recurrent hospitalizations for profound lactic acidosis of unknown etiology presented to the Emergency Department (ED) with altered sensorium and shortness of breath. The patient had been discharged from the hospital 12 hours earlier after management of a similar illness. The current presentation was her sixth hospital admission within the previous six months. All prior presentations shared similar symptoms and laboratory findings: acute onset altered mental status, slurred speech, unsteady gait, tachycardia, and tachypnea in the setting of leukocytosis, acute kidney injury, profound lactic acidosis (ranging from $10-30 \mathrm{mmol} / \mathrm{L}$ ), high anion gap (often greater than $30 \mathrm{mEq} / \mathrm{L}$ ), normal osmolal gap (5-10 mOsm/kg), and negative toxicology studies. Although EG ingestion had been considered during many of these prior admissions, the patient's uniformly low osmolal gap, normal urinalysis, marked lactic acidosis, and negative blood volatile studies had prompted broadening of the differential and extensive evaluation for a suspected mitochondrial, infectious, or inherited metabolic disorder. Despite repeated and exhaustive evaluations, no definitive toxic, infectious, or metabolic etiology had been identified.

On arrival to the ED, the patient was afebrile $\left(36.3^{\circ} \mathrm{C}\right)$, tachycardic $(114$ beats per minute), and tachypneic ( 22 breaths per minute) with an oxygen saturation of $100 \%$ while breathing ambient air. Her initial laboratory studies were remarkable for a white blood cell count of $18.9 \mathrm{~K} / \mathrm{uL}$ (ref: 4-10.9 K/uL), pH 7.13 (ref: 7.35-7.45), anion gap $35 \mathrm{mEq} / \mathrm{L}$ (ref: $3-15 \mathrm{mEq} / \mathrm{L}$ ), lactic acid 14 mmol/L (ref: 0.5-2.0 mmol/L), osmolal gap $5 \mathrm{mOsm} / \mathrm{L}$ (ref: $<10 \mathrm{mOsm} / \mathrm{L}$ ), normal chemical and microscopic urinalysis, negative serum and urine toxicology studies, and undetectable blood volatile organic compounds. Despite the absence of an osmolal gap, her anion gap and lactic acidosis prompted concern for EG ingestion and fomepizole therapy was prophylactically initiated.

Three hours after her arrival to the ED (15 hours after her most recent hospital discharge), the patient declined with a rapid deterioration in mental status accompanied by acute hypoxemic respiratory failure. She was intubated and admitted to the medical intensive care unit. The first 24 hours of her hospital course were complicated by refractory shock, complete heart block, new non-ST elevation myocardial infarction accompanied by severe systolic heart failure with an estimated left ventricular ejection fraction of $15 \%$, and persistence of the severe anion gap metabolic acidosis (30 mEq/L). Hemodialysis was initiated. On hospital day 2 , she became febrile to $40.3{ }^{\circ} \mathrm{C}$ with worsening hyperkalemia and hyperphosphatemia despite hemodialysis. The neurologic examination declined from Glasgow Coma Scale (GCS) 15 with no focal 


\section{Cureus}

neurologic deficits on admission to GCS 3 with absent brainstem reflexes. Repeat noncontrast head computed tomography acquired 36 hours after presentation revealed interval development of bilateral basal ganglia and midbrain hypoattenuation, a nonspecific radiographic finding that can arise secondary to infectious etiologies, toxic insults, inflammatory conditions, or metabolic disorders (Figure 1) [14].

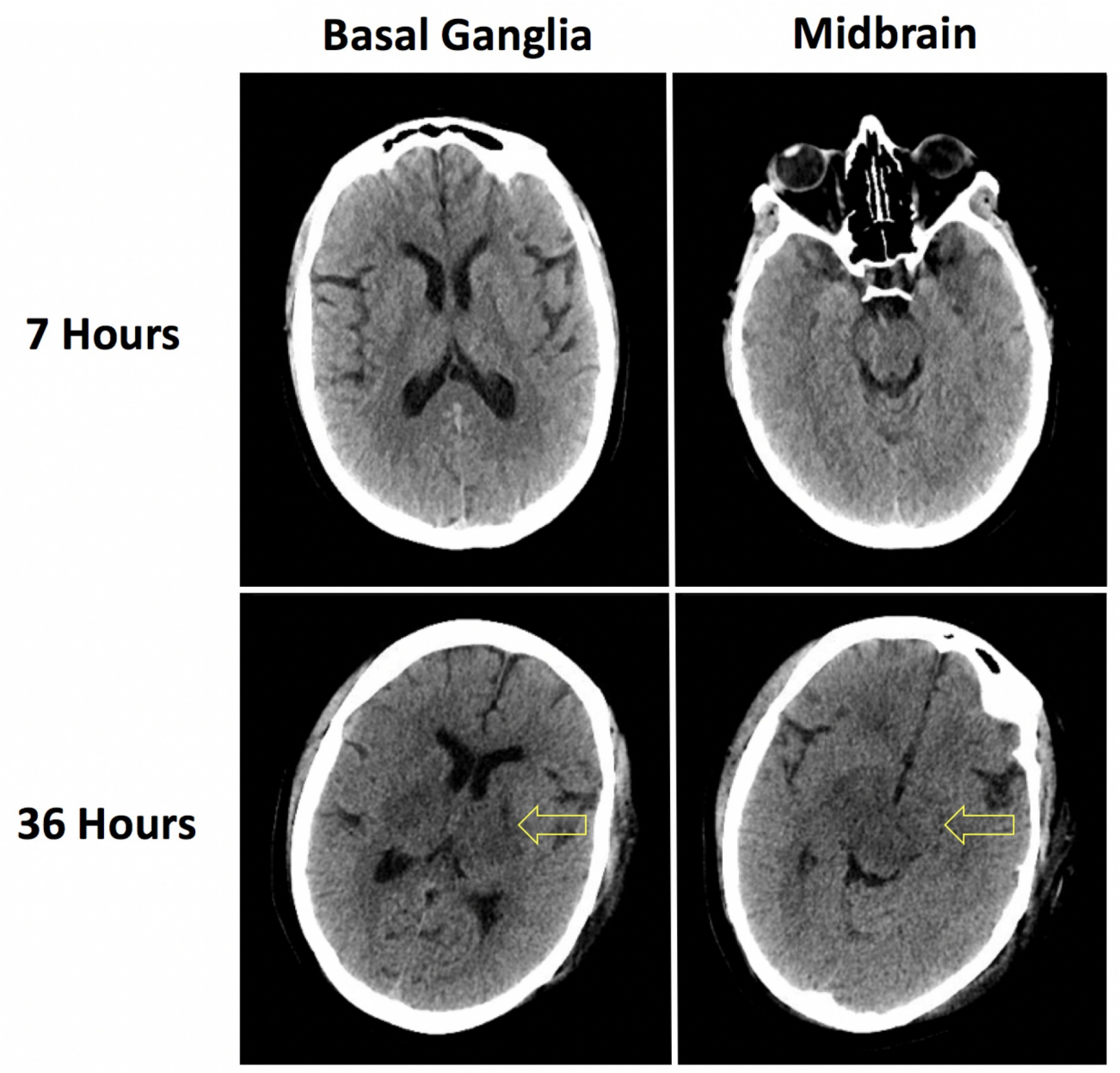

\section{FIGURE 1: Noncontrast computed tomography imaging}

Noncontrast head computed tomography imaging acquired 7 hours and 36 hours after presentation revealing interval development of basal ganglia and midbrain hypoattenuation. Although nonspecific, these radiographic findings are consistent with ethylene glycol toxicity.

In accordance with the family's wishes, the supportive care was withdrawn 72 hours after the admission. All the final culture results and toxicology studies returned negative. At the request of the family, an autopsy was performed and the case was referred to the medical examiner.

Autopsy revealed extensive intravascular and perivascular oxalate crystal deposition. Intravascular foreign material, consistent with oxalate crystals, was identified throughout the renal parenchyma and brain. These findings are pathognomonic for EG ingestion (Figure 2). 


\section{Cureus}
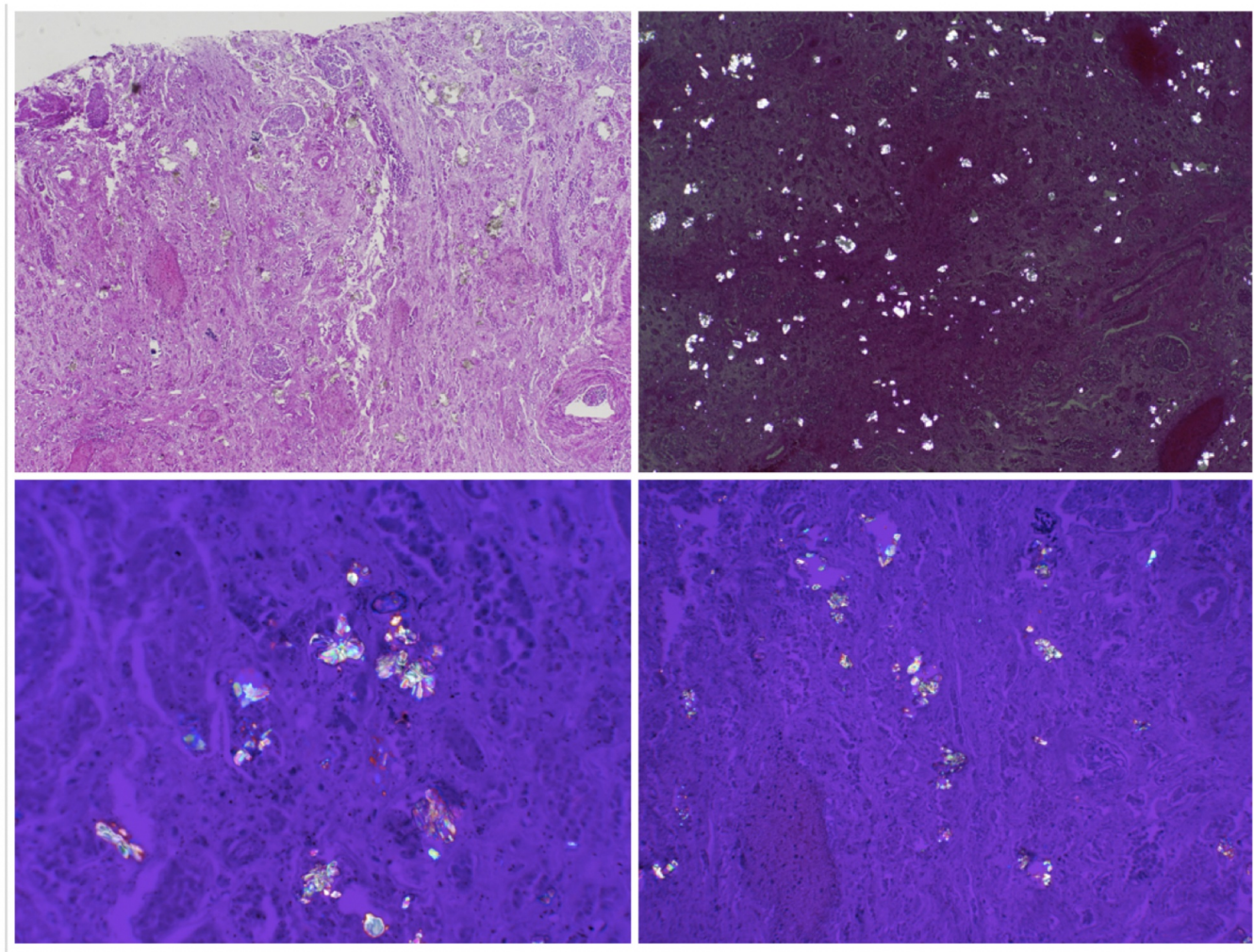

\section{FIGURE 2: Autopsy findings}

Photomicrographs showing diffuse calcium oxalate deposition throughout the renal parenchyma, a finding pathognomonic for ethylene glycol poisoning.

\section{Discussion}

EG toxicity characteristically presents as a severe metabolic acidosis with a high anion gap (27.8 +/- $12.4 \mathrm{mEq} / \mathrm{L})$, elevated osmolal gap (33.3 +/- $5.99 \mathrm{mOsm} / \mathrm{L})$ and large mean base deficit (18.6 $+/-10.9 \mathrm{mEq} / \mathrm{L}$ ) [15]. A significantly elevated osmolal gap is highly specific but poorly sensitive for EG poisoning [16-17]. Pure EG is rapidly metabolized and the likelihood of detecting an osmolal gap decreases with time following ingestion (Figure 3). It has been demonstrated in vivo that the enzymatic efficiency of ADH in metabolizing EG monohydrate and eliminating the osmolal gap may be accelerated in the setting of multiple past toxic alcohol exposures [13]. The present case is unique because of the documented 12-hour interval between hospital discharge and readmission; the patient's clinical history suggests that it was only several hours prior to collection of admission laboratory studies that lethal ingestion of EG occurred. The absence of an osmolal gap in this case supports the concept that the efficiency of toxic alcohol metabolism can be significantly enhanced in patients with past exposures. 


\section{Cureus}

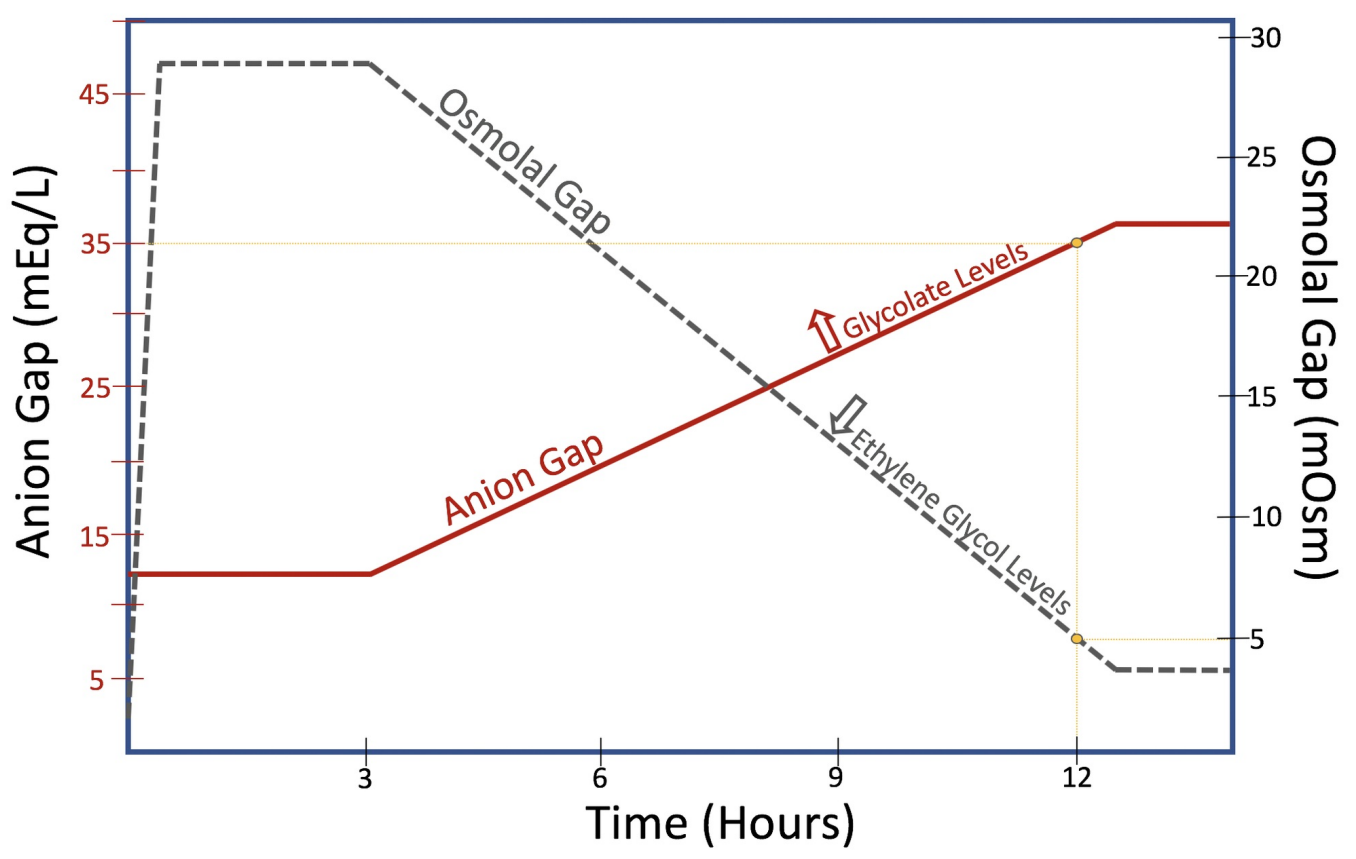

\section{FIGURE 3: Osmolal gap to anion gap conversion}

Visual schematic depicting the relationship between the osmolal gap (solid line) and anion gap. (dashed line). As ethylene glycol is metabolized into toxic, non-osmotic metabolites (i.e. glycolate), the osmolal gap decreases and the anion gap begins to rise.

Artefactual lactic acidosis is an additional complicating factor in the diagnosis of EG poisoning. Glycolate, one of the primary metabolites of EG, is structurally similar to lactate and high circulating levels of glycolate following the EG consumption can be falsely interpreted as a lactic acidosis [11]. Additionally, glycolate is a tasteless and odorless powder that is marketed over the counter as a primary ingredient in many cosmetic products and skin emollients. A toxic ingestion of glycolate would be undetectable in the osmolal gap and virtually indistinguishable from a delayed presentation of the EG poisoning. An understanding of the association between glycolate, artefactual lactate and EG is critical for formulating the complete differential and reaching an early and accurate diagnosis.

The case described in this report is unique in that a lethal EG exposure with a normal osmolal gap confirmed within 12 hours of the toxic ingestion has not been previously reported. A normal osmolal gap has been found only in a small subset of patients suffering from severe EG intoxication, and all of these cases involve patients who presented more than 12 hours following ingestion (Table 1) [2, 18-19]. 


\section{Cureus}

\begin{tabular}{|l|l|l|l|}
\hline Patient & Anion Gap (mEq/L) & Osmolal Gap (mOsm) & Time: Consumption to Presentation \\
\hline 71yo Male [19] & 40 & 4 & $>24$ hours \\
35yo Male [19] & 48 & 7 & $>24$ hours \\
42yo Male [6] & 42 & 9 & 48 hours \\
49yo Male [20] & 32 & 4 & 10 days \\
23yo Male [18] & 25 & 7.2 & $12-24$ hours \\
\hline
\end{tabular}

TABLE 1: Reported cases of ethylene glycol toxicity with a normal osmolal gap

Notably, the only documented case of EG exposure with a normal osmolal gap presenting close to 12 hours from the EG ingestion involved a patient who similarly had a history of prior toxic alcohol consumption [18].

\section{Conclusions}

For patients with a history of toxic alcohol abuse, the duration of time required to convert EG into its non-osmotically active toxic metabolites can be significantly decreased. In this subset of patients, the osmolal gap will be within normal limits shortly after ingestion and an accelerated progression through the stages of EG toxicity can occur. Severe lactic acidosis in the setting of a normal osmolal gap in a chronic alcoholic patient should be interpreted with caution, as this can be found in patients with chronic EG exposure.

\section{Additional Information}

\section{Disclosures}

Human subjects: Consent was obtained by all participants in this study. Conflicts of interest: In compliance with the ICMJE uniform disclosure form, all authors declare the following:

Payment/services info: All authors have declared that no financial support was received from any organization for the submitted work. Financial relationships: All authors have declared that they have no financial relationships at present or within the previous three years with any organizations that might have an interest in the submitted work. Other relationships: All authors have declared that there are no other relationships or activities that could appear to have influenced the submitted work.

\section{References}

1. Hlozek T, Bursova M, Cabalaa R: Fast determination of ethylene glycol, 1,2-propylene glycol and glycolic acid in blood serum and urine for emergency and clinical toxicology by GC-FID. Talanta. 2014, 130:470-474. 10.1016/j.talanta.2014.07.020

2. Ammar KA, Heckerling PS: Ethylene glycol poisoning with a normal anion gap caused by concurrent ethanol ingestion: importance of the osmolal gap. Am J Kidney Dis. 1996, 27:130133.

3. Moreau CL, Kerns W, Tomaszewski CA, et al.: Glycolate kinetics and hemodialysis clearance in ethylene glycol poisoning. META Study Group. J Toxicol Clin Toxicol. 1998, 36:659-666.

4. Glaser DS: Utility of the serum osmol gap in the diagnosis of methanol or ethylene glycol ingestion. Ann Emerg Med. 1996, 27:343-346. 10.1016/S0196-0644(96)70271-8

5. Mycyk M, Aks S: A visual schematic for clarifying the temporal relationship between the anion 
and osmol gaps in toxic alcohol poisoning. Am J Emerg Med. 2003, 21:3.

6. Arora A: The 'gap' in the 'plasma osmolar gap'. BMJ Case Rep. 2013, 10.1136/bcr-2013-200250

7. Long H, Nelson LS, Hoffman RS: A rapid qualitative test for suspected ethylene glycol poisoning. Acad Emerg Med. 2008, 15:688-690.

8. Church AS, Witting MD: Laboratory testing in ethanol, methanol, ethylene glycol, and isopropanol toxicities. J Emerg Med. 1997, 15:687-692.

9. Kraut JA: Diagnosis of toxic alcohols: limitations of present methods . Clin Toxicol. 2015, 53:589-595. 10.3109/15563650.2015.1056880

10. Jacobsen D, Bredesen JE, Eide I, Ostborg J: Anion and osmolal gaps in the diagnosis of methanol and ethylene glycol poisoning. Acta Med Scand. 1982, 212:17-20.

11. Pernet P, Beneteau-Burnat B, Vaubourdolle M, Maury E, Offenstadt G: False elevation of blood lactate reveals ethylene glycol poisoning. Am J Emerg Med. 2009, 27:132-131.

10.1016/j.ajem.2008.04.029

12. Cederbaum AI: Alcohol metabolism. Clin Liver Dis. 2012, 16:667-685. 10.1016/j.cld.2012.08.002

13. Kawamoto T, Matsuno K, Kayama F, Hirai M, Arashidani K, Yoshikawa M, Kodama Y: Effect of ethylene glycol monomethyl ether and diethylene glycol monomethyl ether on hepatic metabolizing enzymes. Toxicology. 1990, 62:265-274.

14. Hegde AN, Mohan S, Lath N, Lim CC: Differential diagnosis for bilateral abnormalities of the basal ganglia and thalamus. Radiographics. 2011, 31:5-30. 10.1148/rg.311105041

15. Latus J, Kimmel M, Alscher MD, Braun N: Ethylene glycol poisoning: a rare but lifethreatening cause of metabolic acidosis-a single-centre experience. Clin Kidney J. 2012, 5:120-123. 10.1093/ckj/sfs009

16. Krasowski MD, Wilcoxon RM, Miron J: A retrospective analysis of glycol and toxic alcohol ingestion: utility of anion and osmolal gaps. BMC Clin Pathol. 2012, 12:1. 10.1186/1472-6890$12-1$

17. Bartlett D: Understanding the anion and osmolal gaps laboratory values: what they are and how to use them. J Emerg Nurs. 2005, 31:3.

18. Steinhart B: Case report: severe ethylene glycol intoxication with normal osmolal gap: "a chilling thought". J Emerg Med. 1990, 8:3.

19. Darchy B, Abruzzese L, Pitiot O, Figueredo B, Domart Y: Delayed admission for ethylene glycol poisoning: lack of elevated serum osmol gap. Intensive Care Med. 1999, 25:859-861.

20. Ting SM, Ching I, Nair H, Langman G, Suresh V, Temple RM: Early and late presentations of ethylene glycol poisoning. Am J Kidney Dis. 2009, 53:1091-7. 